\title{
Calzado laboral y actuación podológica
}

\author{
Occupational shoes and podiatric actuation
}

\author{
Mario Mencía Fernández \\ Diplomado en Enfermería.
}

\begin{abstract}
Correspondencia:
Mario Mencía Fernández.

C/ Puente Nuevo $n^{\circ} 184^{\circ} \mathrm{B}$

45600 Talavera de la Reina

Toledo
\end{abstract}

Fecha de recepción: 10 septiembre 2009

Fecha de aceptación: 2 febrero 2010

El autor declara no tener ningún tipo de interés económico o comercial.

\section{RESUMEN}

En el siguiente estudio, tras hacer una presunción sobre el uso del calzado laboral y la patología derivada del uso y el abuso del mismo, el uso del calzado laboral a tenor de evitar traumatismos directos en el pie y la unidad ungueal y otros riesgos, tanto químicos como biológicos y ambientales, también es un factor desencadenante en la producción de la patología podológica.

La falta de información que se lleva a cabo en las empresas, en parte por falta de podólogos experimentados en la materia que instruyan a la población laboral en cada empresa, y en parte por falta de interés por el colectivo podológico en abordar la salud podológica laboral como una más de sus funciones, de las cuales podemos producir un importante aumento cualitativo y cuantitativo en la eficacia y eficiencia de los trabajadores y contribuir a introducir el colectivo podológico como parte de los servicios de prevención en las empresas, donde no se evidencia nuestra presencia, también sería importante reseñar la poca coordinación entre las empresas manufactureras de calzados y el muy beneficioso aporte de la podología a estas industrias.

A través del estudio realizado a distintos trabajadores de distintas empresas y sectores, he seleccionado información sobre la presencia de patología podológica por uso de calzado laboral y también información sobre la confortabilidad del mismo para delimitar cuales deberían de ser las funciones de la podología en el ámbito laboral.

Palabras clave: Calzado laboral, patología podológica, empresas.

\begin{abstract}
In the next study, before make a presumption about the use and abuse of work shoe and the podiatric pathology, the use of work shoe to prevent of the direct trauma in the foot and nail and other risks, so much chemical risks and biological and environmental risks, as well is a causing factor in the production of the podiatric pathology.

The abscense of information in the business, in part for the abscense of experimented podiatrics in this field that learning to the laboral population in the firm, as well for the staff of interest for the podiatric group in the treatment of the podiatric work health as a of the role as podiatry, derive of this functions to produce a important rise as qualitative as quantitative in the efficacy and the efficiency of the workers in the firms, where there isn't our presence, also it is very important to review the little coordination between the firms manufacturer of shoes and the best contribution that the podiatry could to make in these firms.
\end{abstract}


As well as trough the study done to many workers of many firms and areas, I've choosen information about the presence or abscense of podiatric pathology for use of the occupational footwear and also about the confortability for to define who will must to be the duties of podiatric medical in the work area.

Keywords: Work shoes, podiatric pathology, firms.

Sumario: 1. Introducción. 2. Objetivos. 3. Material y método. 4. Discusión. 5. Conclusión. 6. Bibliografía e internetgrafía.

\section{Referencia normalizada:}

Mencia Fernández, M. Calzado laboral y actuación podológica. Revista Internacional de Ciencias Podológicas 2011; 5 (1): 9-19. 


\section{INTRODUCCIÓN}

Debido a la exigencia de la normativa actual en cuanto a la seguridad laboral en el trabajo, de los equipos de protección individual (epi's, norma EN-344,EN-345,EN-346,EN-347)2, hoy en día el calzado laboral como desde sus comienzos tiene la función de defender al pie y la pierna de las agresiones producidas en el medio laboral como resultado del trabajo realizado.

Hoy en día el calzado laboral llega a su exponente máximo ya que disponemos en el mercado de una serie de materiales que podrían hacer del calzado laboral no solo un calzado protector de lesiones en el pie ,sino que también y debido a este abanico de posibilidades se debería revisar el diseño y la forma de los distintos calzados, tanto de seguridad como de protección y ocupacionales, así como los materiales que se emplean en su fabricación para evitar lesiones debidas al uso del calzado laboral, aunque ya se han patentado algunos calzados ocupacionales con punteras de fibra de vidrio y composites ${ }^{8}$, para evitar lesiones en los dedos, a la vez que se rebaja el peso del calzado.

La mayoría de los estudios que he revisado en la bibliografía se centran en problemas con las suelas de los calzados, suelas antideslizante y anticaída, suelas antiestáticas, para evitar riesgos eléctricos ${ }^{4}$, otros estudios hablan del confort $^{17}$, otros hacen referencia al calzado de algunos grupos laborales como los leñadores o bomberos, haciendo mención a la bota que deben llevar y al diseño necesario para evitar lesiones en pie y pierna ${ }^{11,12}$, otros hacen mención a revisiones bibliográficas sobre el papel que desempeña el podólogo en la empresa, haciendo referencia a la posible inclusión de los servicios de Medicina laboral, del podólogo ${ }^{3}$.

Según el Ministerio de Trabajo e Inmigración $^{1}$, en 2007, se produjeron en España 255.353 accidentes que cursaron baja laboral lo que supone un alto coste económico y personal. Todos estos accidentes se podrían evitar usando un calzado laboral adecuado a cada tipo de trabajador y dependiendo del tipo de actividad a que se dedique. Para llevar a cabo este estudio hemos utilizado como descriptores: "safety shoes", "Safety footwear" buscando en Medline, Pubmed y Science Direct encontrando un total de 85 referencias bibliográficas.

Mediante un estudio realizado a trabajadores de distintas empresas, dedicadas a actividades muy distintas, he intentado obtener unos resultados que me hablarán no solo de la confortabilidad del calzado laboral, sino también y yendo un poco más lejos de las lesiones producidas por el calzado laboral.

\section{OBJETIVOS}

Mediante el siguiente estudio mis objetivos son los siguientes:

- Determinar los problemas podológicos asociados al uso o sobreuso del calzado laboral.

- Hacer partícipe al podólogo de la salud laboral del trabajador.

\section{MATERIAL Y MÉTODO}

Se realizó una revisión bibliográfica de las bases de datos más frecuentes: Medline, Pubmed y Science Direct desde el año 1953 hasta la actualidad. Introducí como descriptores: "safety shoes" y "safety footwear". No se valoraron los estudios en cuyo abstract no incluían el calzado laboral como tema de estudio.

Luego posteriormente he realizado un estudio multicéntrico, descriptivo y prospectivo de octubre de 2008 a marzo de 2009, a 30 trabajadores de distintas empresas dedicadas a las telecomunicaciones, sanidad, construcción y afines, hostelería y otras, utilizando un cuestionario de preguntas (Anexo I), mediante entrevista "face to face"; con el fin de evidenciar los objetivos del estudio. Como criterio de inclusión, solo hay uno y es el siguiente: trabajadores de cualquier actividad que en su cometido diario utilicen calzado laboral, de seguridad o de protección.

\section{RESULTADOS}

Según el estudio realizado a distintos trabajadores de empresas tan variadas como la sanidad, las telecomunicaciones, la hostelería, la construcción y afines y otras en las cuales se utiliza calzado laboral para realizar el co- 
metido diario, se han obtenido los siguientes resultados:

De todos los encuestados un $47 \%$ corresponde al sector telecomunicaciones, un $20 \%$ a la sanidad, un $13 \%$ a la construcción y afines, y un $10 \%$ tanto para la hostelería como para otros (Figura 1).

Con respecto al tipo de calzado laboral que emplean estos en su cometido diario, encontramos, que un $63 \%$ de los encuestados utilizan calzado laboral con refuerzos, un $17 \%$ utilizan zueco sin aditamento posterior, sobre todo en el sector sanidad, y un $10 \%$ tanto para los que utilizan calzado sanitario cerrado y para quien utiliza calzado laboral sin refuerzos (Figura 2).

El tiempo que trabajan a la semana, se cuantifica en un $3 \%$ para los que trabajan $<35$ horas, un $7 \%$ para los que trabajan $35 \mathrm{~h}$, un $40 \%$ para los que trabajan $>42 \mathrm{~h}$ y un $50 \%$ para aquellos que trabajan entre $35 \mathrm{y} 42 \mathrm{~h}$ semanales (Figura 3).

La incidencia patológica previa o actual la cuantificamos en un 2\% para las patologías que cursan con engrosamiento de la lámina un-

\section{Distribución sectorial}

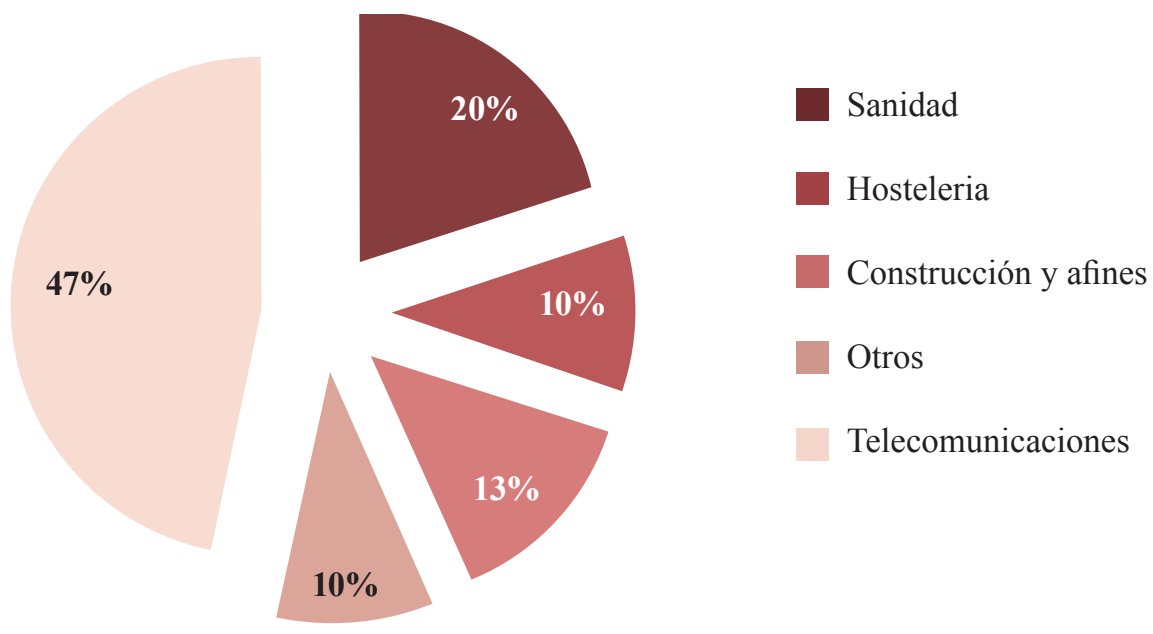

Figura 1. Distribución por sectores de los trabajadores de empresas como la sanidad, las telecomunicaciones, la hostelería, la construcción y otras en las cuales se utiliza calzado laboral.

\section{Tipo de calzado laboral}

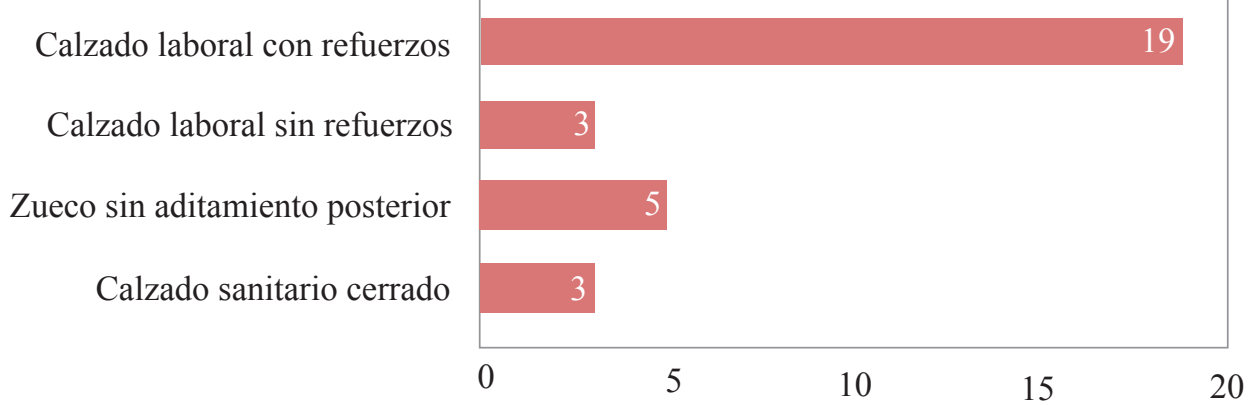

Figura 2. Tipo de calzado laboral que se emplea diariamente. 
gueal, un $17 \%$ en la presencia de enclavamiento ungueal, otro $2 \%$ en la aparición de estrías en las uñas, un $27 \%$ en la presencia de callosidades y helomas, un $18 \%$ en la aparición de sequedad en el pie, un $10 \%$ en la presencia de estrías en el talón, $3 \%$ en los cambios en la curvatura de las uñas, un $8 \%$ en la coloración de las uñas, pie plano $5 \%$, pie pronado $5 \%$ y pie cavo un $3 \%$. Tener en cuenta que esta pregunta es de tipo multirespuesta (Figura 4).

\section{Tiempo de trabajo a la semana}

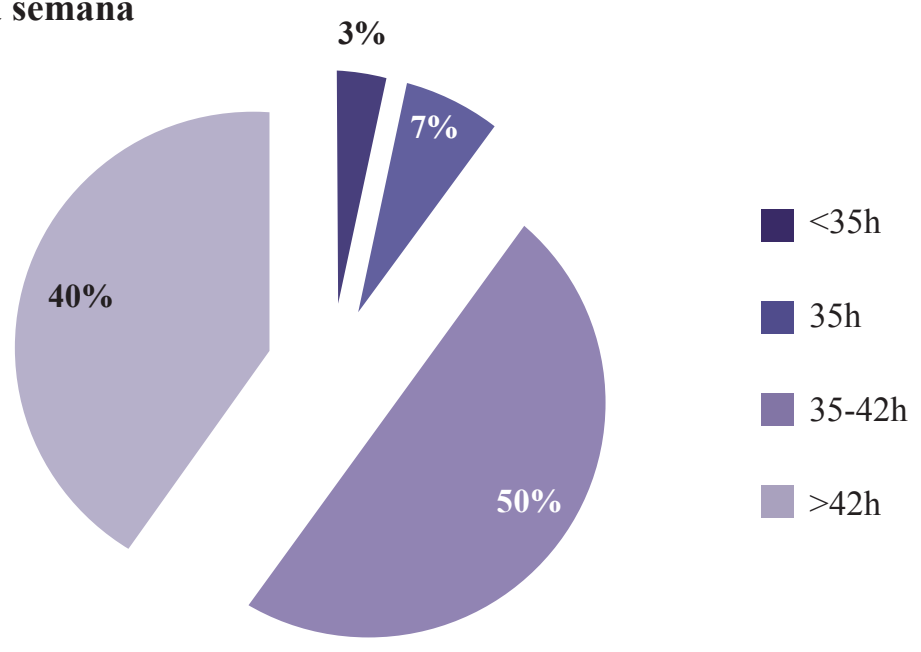

Figura 3. Distribución porcentuada del tiempo de trabajo en horas a la semana.

\section{Incidencia de la patología podológica}

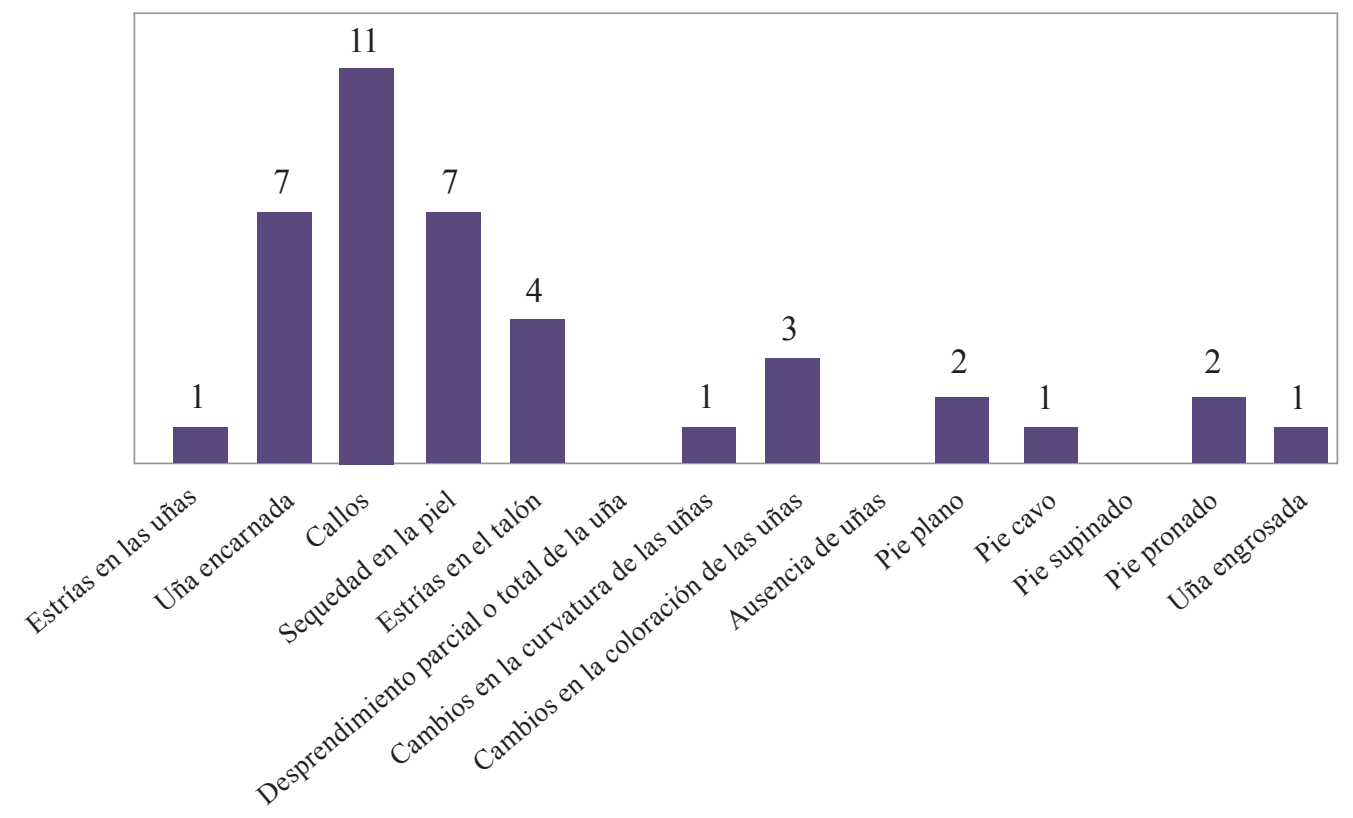

Figura 4. Incidencia de la patología que presentan los trabajadores. 
Sobre el sufrimiento infringido por el uso del calzado laboral he cuantificado : un $21 \%$ para los que no experimentaron sufrimiento durante ni después del uso del calzado laboral, un $25 \%$ para los que si experimentaron algún tipo de sufrimiento, un $9 \%$ para los que contestaron que a veces, un $29 \%$ para los que contestaron que sufrieron durante el uso y un $21 \%$ para los que contestaron que después. Estos resultados pueden estar compartidos ya que el que sufre durante el uso, también puede sufrir después y viceversa, o solamente padecer durante el uso o después del uso (Figura 5).

A la pregunta sobre enfermedades de las uñas o de los pies derivados del uso del calzado laboral actuales, se evidencia un $20 \%$ de positivos y un $80 \%$ de negativos. A la pregunta sobre la existencia de tratamiento podologico previo o actual contestaron un $23 \%$ positivamente y un $77 \%$ negativamente (Figura 6).

\section{Sufrimiento por el uso del calzado}

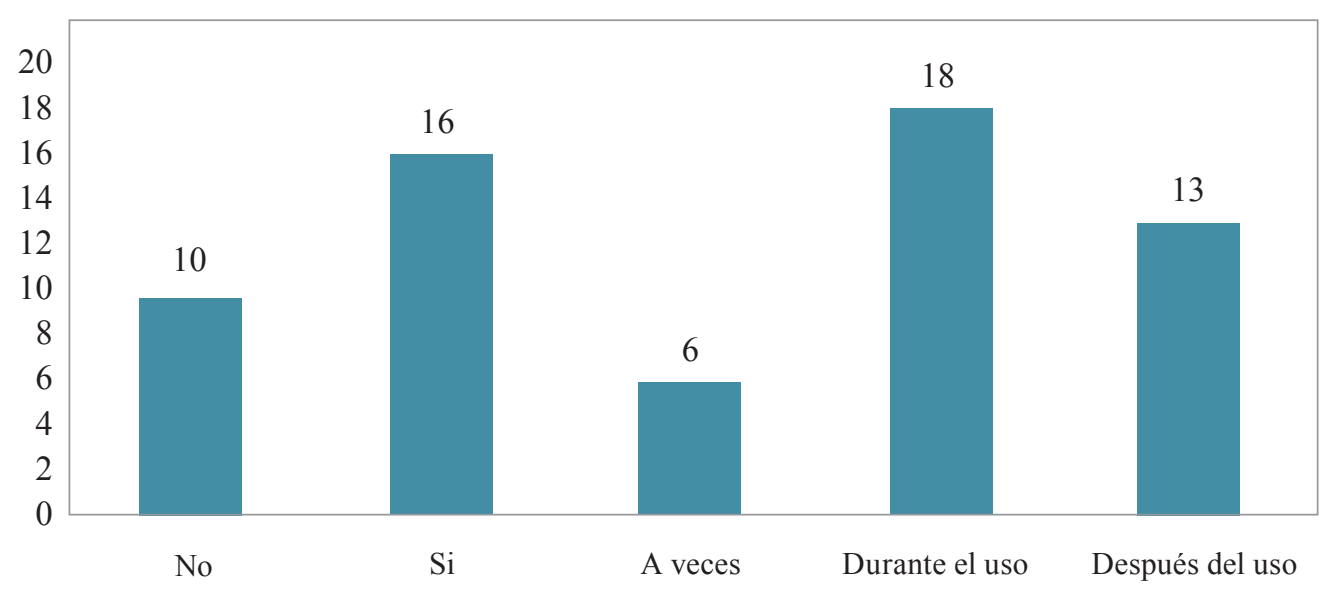

Figura 5. sufrimiento infringido por el uso del calzado laboral.

Existencia de $\mathrm{tt}^{\mathrm{o}}$ podologico previo o actual dvdo. uso calzado laboral

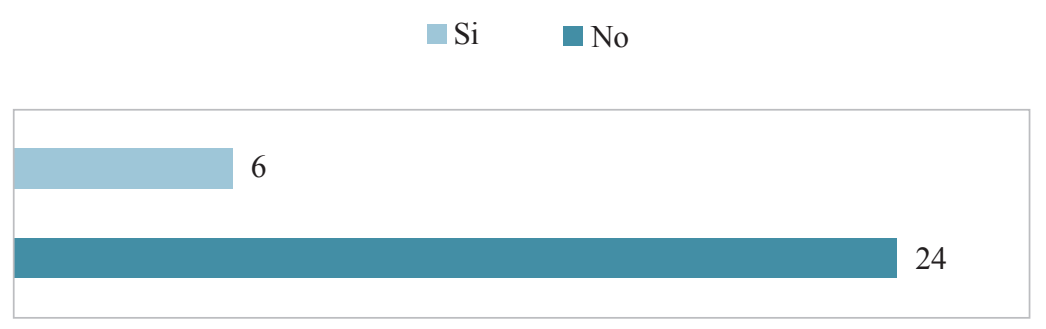

Figura 6. Enfermedades de las uñas o de los pies derivados del uso del calzado laboral. 
A cerca de la pregunta que hace referencia al peso del calzado laboral, los trabajadores contestaron $1 \mathrm{~kg}$ el $93 \%$ y $2 \mathrm{~kg}$ el $7 \%$, a la respuesta de $500 \mathrm{~g}$ no contesto nadie (Figura 7).

A la pregunta sobre si realizaría cambios en su calzado laboral y si es en caso afirmativo entonces se le pregunta al trabajador el qué, en una pregunta abierta, donde he resumido las respuestas entre la confortabilidad, el sistema de cierre, la suela, la ventilación y el material de la puntera. Un $27 \%$ contestó negativamente, haciéndolo sensiblemente un $73 \%$ positivamente (Figura 8).

Del $73 \%$ que contestó positivamente, se le pidió que diera una contestación abierta explicando en que creía que debería variar su calzado laboral y contestaron con las respuestas mencionadas anteriormente.

Un $22 \%$ de las respuestas referían una mejor confortabilidad, un $18 \%$ referían tener mucho

\section{Peso estimado del calzado laboral}

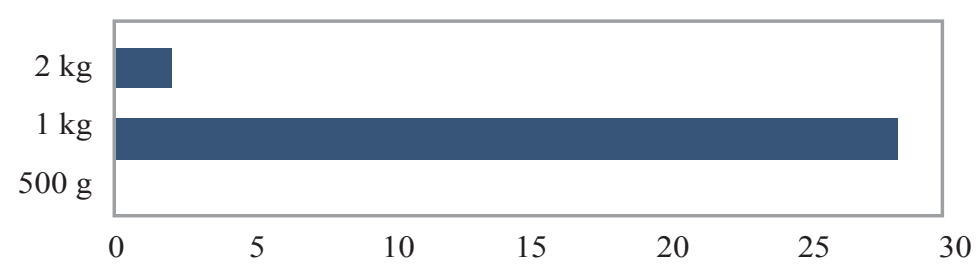

Figura 7. Peso del calzado laboral.

\section{¿Modificaría su calzado laboral?}

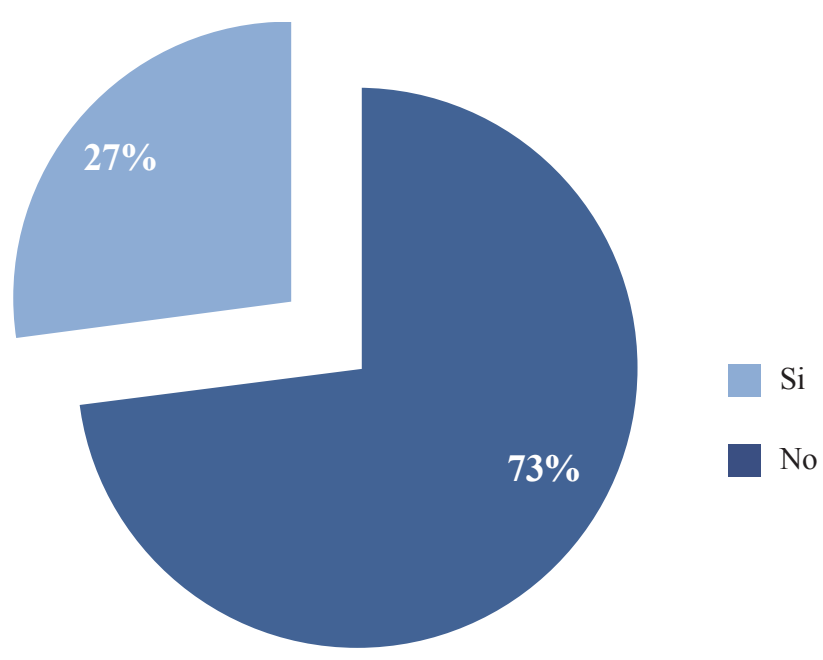

Figura 8. Porcentaje que realizarían cambios en su calzado laboral. 
peso, otro $18 \%$ que decían quejarse del material de la puntera, un $15 \%$ hacen referencia a la excesiva dureza del calzado, un $12 \%$ a las suelas, un $9 \%$ hablan del sistema de cierre y un $6 \%$ de la ventilación (Figura 9).

Sobre la pregunta sobre la seguridad que ofrece el calzado laboral, contestaron ante la misma positivamente un $83 \%$ y negativamente un $17 \%$.

Sobre la pregunta sobre la intervención podológica en el desarrollo y diseño del calzado laboral. Contestaron positivamente un $93 \%$ frente a un 7\% que contestó que le daba igual, negativamente no contestó nadie (Figura 10).

\section{Cambios que realizaría en su calzado laboral}

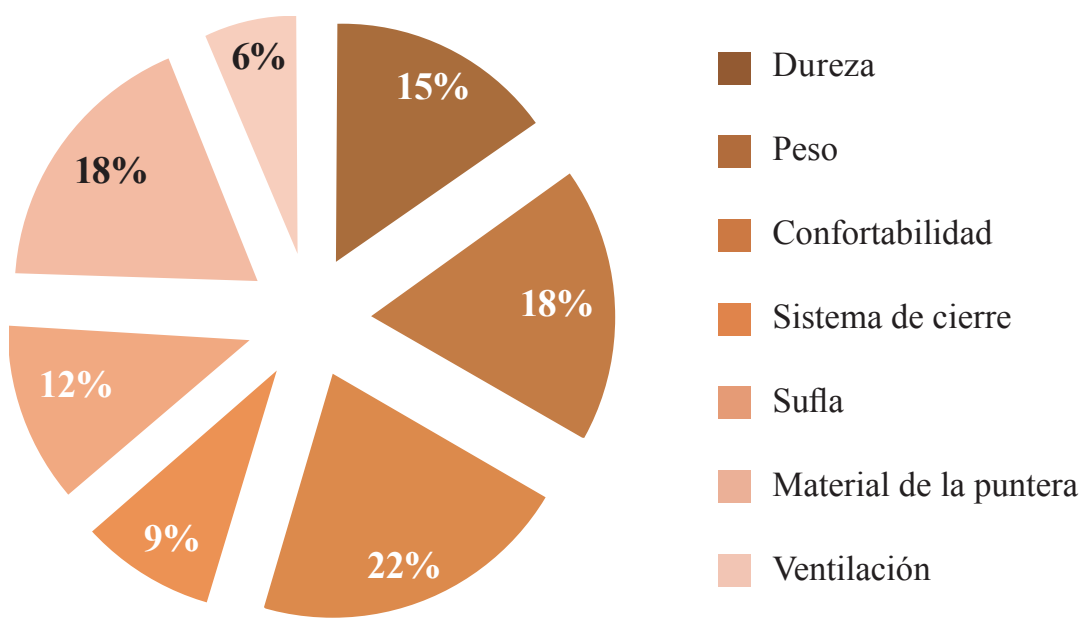

Figura 9. Cambios que deberían realizarse en el calzado laboral.

\section{Percepción del podólogo en la salud laboral}

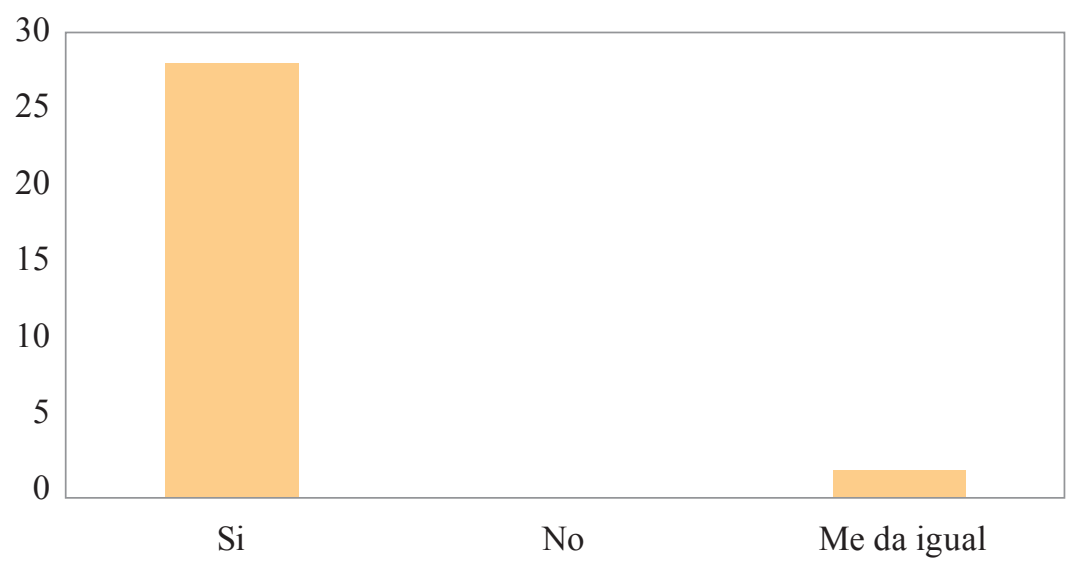

Figura 10. Opinión sobre la posible intervención podológica en el desarrollo y diseño del calzado laboral. 


\section{DISCUSIÓN}

Como vemos la presencia del uso de calzado laboral con refuerzos, es decir con refuerzo en la puntera, e incluso en ocasiones en retropié, es cada vez más habitual a la hora de trabajar en cualquier tipo de empleo, aunque curiosamente en la sanidad es donde menos se evidencia este tipo de calzado, donde se sigue utilizando el zueco sin aditamento posterior, y el calzado sanitario cerrado.

Dentro del estudio realizado la edad promedio ha sido entre 20 y 40 años dentro de los 16 a 64 años que escogí para realizar la encuesta, puesto que esta es la edad de la población activa en España.

Dentro de la muestra de trabajadores elegida se cogieron más individuos del sector telecomunicaciones por una cuestión que escapa al entrevistador, no tiene nada que ver con que fuera mi pretensión que esto fuera así, lo lógico a la hora de escoger una muestra de distintos trabajadores es elegir aproximadamente el mismo número de personas de cada trabajo, pero no pudo ser así, por lo cual creo es conveniente remarcar este punto a propósito de la pregunta 4.

Como hemos podido observar hay un creciente número de personas que ya utilizan calzado laboral con refuerzos en sus trabajos y da igual a lo que se dediquen, con lo cual se evidencia que en las empresas se toman cada vez más en serio la salud del trabajador, ya que va a favor de la empresa.

La muestra analizada trabaja una media de 35-42h, siguiéndole la franja de individuos de $>42 \mathrm{~h}$ muy de cerca, lo cual es significativo a la hora de analizar la posible aparición de patología podológica ya que a mayor tiempo de trabajo mayor incidencia de patología podológica.

Dentro de las patologías podológicas más frecuentes encontramos que las callosidades y helomas, la sequedad en la piel, así como la onicocriptosis y las estrías en el talón son las patologías podológicas más frecuentes derivadas del uso del calzado laboral, lo cual debe hacernos replantearnos a los podólogos la posible intervención en el diseño del calzado laboral.

Ante la pregunta $n^{\circ} 8$, sobre la presencia de daño producido por el calzado laboral obser- vamos que un $25 \%$ contestaron positivamente lo que nos indica que un cuartil de la muestra si ha sufrido daño, si a esto añadimos el 6\% de los que contestaron que a veces, estamos ante un 31\% que representa casi un tercio de la muestra lo cual es bastante significativo y deberíamos de plantearnos si se diseñan bien los calzados laborales.

Ante la pregunta $n^{\circ} 9$ se evidencia un $20 \%$ de la muestra que presenta actualmente daño por el calzado utilizado en su actividad diaria, bastante representativo ya que es un quinto de la muestra.

Ante la pregunta $n^{\circ} 10$ encontramos un tímido $23 \%$ que han estado en tratamiento previo o actual por enfermedades de las uñas o de la piel derivado del uso del calzado laboral, lo cual nos orienta sobre la intervención podológica, que cada vez va siendo más importante en las poblaciones laborales.

Ante la pregunta $n^{\circ} 11$ curiosamente un 93\% contestó que su calzado laboral pesaba $\sim 1 \mathrm{~kg}$ que es lo que suelen pesar un par de calzados de seguridad, pero luego en la siguiente pregunta cuando se les pregunta sobre si modificarían su calzado laboral, un 18\%, del 73\% que contestó positivamente, indicaba que si cambiase algo sería el peso, lo cual sería una cuestión importante a la hora de plantearse la distribución de peso que recibe el calzado, porque esta sensación de peso excesiva puede venir dada por una mala distribución de los materiales que se utilizan en la elaboración del calzado laboral, pudiendo ocasionar patologías típicas del antepié o del retropié.

Llama la atención también que del 73\% que contestó que sí modificaría su calzado laboral, es un número lo bastante alto como para hacernos reflexionar al colectivo podológico así como a la industria sobre qué es lo que estamos haciendo mal, las principales quejas o posibles modificaciones, hacen referencia a la confortabilidad (22\%), El material de la puntera y el peso (18\%), así como la dureza $(15 \%)$ y las suelas (12\%) dentro de los datos más representativos.

Luego curiosamente los individuos en la pregunta 13 indican en un $83 \%$ que les parece que su calzado les confiere la seguridad inherente a su puesto de trabajo, lo cual se con- 
tradice un poco con los resultados anteriores, pero no obstante nada desechables.

Ante la pregunta 14 se ha mostrado un resultado del $93 \%$ de positivos, lo cual indica que dicho de boca de los trabajadores, sería bueno que el colectivo podológico se planteara la creciente necesidad de incluir al podólogo dentro de la salud laboral, pudiendo así establecer programas de prevención podológica, favoreciendo al diseño del calzado laboral y a la elección de uno u otro calzado laboral según el tipo de pie ante el cual nos encontremos, así como la utilización de tratamientos ortopodológicos diseñados por el podólogo para evitar la aparición de las patologías ya mencionadas.

\section{CONCLUSIÓN}

Creo que con este estudio se evidencia que debido a la utilización del calzado laboral ni que decir tiene que aunque se evitan lesiones más importantes de los pies y el aparato ungueal, también se potencian otras lesiones que aunque son menores pueden ser igualmente in- capacitantes y pueden provocar bajas laborales largas, lo cual va en detrimento del trabajador y de la empresa.

También creo firmemente que el podólogo debería de ser parte principal dentro de los servicios de prevención ajenos de las empresas ya que es una pieza, como hemos visto fundamental, que puede participar en el desarrollo de programas de prevención, en el diseño del calzado, en la elección de uno u otro tipo de calzado laboral, como asesor del calzado más adecuado (podiatric advisor) así como de la recomendación de la utilización de ciertos tratamientos ortopodológicos que mejoren la salud del trabajador.

\section{AGRADECIMIENTOS}

Agradecer a todos los voluntarios que hicieron posible este estudio, a los empleados de la empresa Teletrix Instalaciones S.L., a los trabajadores de la empresa Los Tinajones (Hostelería), y a los empleados del SESCAM, sin los cuales no hubiera sido posible hacer este estudio.

\section{BIBLIOGRAFÍA E INTERNETGRAFÍA}

1. Ministerio de Trabajo e Inmigración. Anuario de estadísticas del ministerio de Trabajo e Inmigración 2007. Disponible en: www.mtas.es/estadisticas/anuario2007/ATE/ate19_top_HTML. htm

2. Artículo 4 Ley 31/1995, de 8 de noviembre de Prevención de Riesgos Laborales. BOE n²69 de 10 de noviembre

3. Instituto Nacional de Seguridad e Higiene en el Trabajo. Guía orientativa para la selección y utilización de EPI, calzado de uso profesional. Disponible en: www.insht.es/InshtWeb/ Contenidos/Documentacion/TextosOnline/Guias/Guias_Orientativas_EPI/Ficheros/calzado_uso_profesional.pdf

4. Alonso Montero C, Chicharro E, Padrós N, Marhuenda D. Calzado de Seguridad y el papel del podólogo en la seguridad laboral. El Peu 2008 , 28(4): 172-178

5. Puigcerver Palau S, González J.C. FAL Calzado de Seguridad S.A., desarrolla una gama innovadora de suelas antideslizantes para calzado de seguridad. Revista de Biomecánica 2006, 45: $23-25$

6. Botana Pazos, O. Calzado de seguridad. Podoscopio 2005; 30: 192-198

7. Comín Clavijo, M. El IBV elabora los pliegos de prescripciones técnicas para la adquisición de calzado ocupacional de Iberia Líneas Aéreas de España S.A.Revista de Biomecánica 2005,43: 23-27

8. Lee SM, Lim TS, Lee DG.Damage tolerance of composite toe cap. Composites structures 2005;67:167-74

9. Martín Pies F. Calzado hospitalario. Revista Española de Podología 1997, VIII (4): 199-201 
10. Boarriza JJ, $2^{\circ}$ Symposium Nacional de Podología, Zaragoza.Consideraciones sobre profilaxis y terapeútica en el pie del trabajador por la incidencia del calzado industrial. Revista Española de Podología 1973; 36: 28-31

11. Pérez Quirós, M. Estudio del calzado laboral de uso más frecuente. Revista Española de Podología 1993; 6: 280-297

12. Farreras Morcillo F. Podología laboral: su importancia social. Revista Española de Podología 1973, 35: 9-14

13. Marr S. Firefighter's safety footwear : an investigation into problems reported by firefighters. J Occup Health Safety- Aust-NZ 1990; 6(4): 297-301

14. Reilly M. Selecting proper safety footwear. Occup Health Saf 2007 Oct; 76(10): 72-77.

15. Kirk P, Parker R. Análisis de la bota de pinchos en la seguridad del leñador. App Erg 1994; 25(2): 106-110

16. Lastras González S, Fernández de la Monja, VI. El médico del trabajo en el control del uso de epi's y los problemas de salud derivados. Medicina y Seguridad del Trabajo: 2008; 212: 21-32

17. González Gómez E. Importancia del podólogo en la medicina de empresa . Revista Española de Podología 1981, 83: 27-33

18. Marr S. Problems asociated with the wearing of safety footwear. J Occup Health Safety-Aust NZ 1991; 7(5):437-9

19. Marr SJ, Quine S. Shoe concerns and foot problems of wearers of safety footwear. Occup Med 1993; 43(2):73-7

20. Nordress R. Epidemiología y bioestadística,Secretos. Edición en español. Madrid: Elsevier España;2006

21. Cobo E, Muñoz P, González JA. Bioestadística para no estadísticos. Bases para interpretar artículos científicos. Barcelona : Elsevier Doyma; 2007 